\title{
Narrative review on Morbus Fabry: diagnosis and management of cardiac manifestations
}

\author{
Aleš Linhart ${ }^{1,2}$, Tomáš Paleček ${ }^{1,2}$ \\ ${ }^{1}$ 2nd Department of Internal Cardiovascular Medicine, General University Hospital, Prague, Czech Republic; ${ }^{2}$ First Faculty of Medicine, Charles \\ University, U Nemocnice 2, 12808 Praha 2, Czech Republic \\ Contributions: (I) Conception and design: None; (II) Administrative support: None; (III) Provision of study materials or patients: None; (IV) \\ Collection and assembly of data: None; (V) Data analysis and interpretation: None; (VI) Manuscript writing: All authors; (VII) Final approval of \\ manuscript: All authors. \\ Correspondence to: Aleš Linhart. 2nd Department of Internal Cardiovascular Medicine, General University Hospital Prague, Czech Republic; First \\ Faculty of Medicine, Charles University, U Nemocnice 2, 12808 Prague 2, Czech Republic. Email: ales.linhart@vfn.cz.
}

\begin{abstract}
Fabry disease (FD) is an X-linked lysosomal storage disorder due to reduced or undetectable $\alpha$-galactosidase A (AGAL-A) enzyme activity caused by pathogenic variants in the AGAL-A gene $(G L A)$. Tissue and organ changes are caused by widespread progressive accumulation of globotriaosylceramide $\left(\mathrm{Gb}_{3}\right)$ and globotriaosylsphingosine $\left(\mathrm{lysoG}_{3}\right)$. The classical form of FD is multisystemic with cutaneous (angiokeratomas), neurological (peripheral neuropathy, premature stroke), renal (proteinuria and renal insufficiency), and cardiac involvement. Later onset variants may be limited to the heart. The objective of this review is to summarize the current knowledge on cardiac manifestations of FD and effects of targeted therapy. Cardiac involvement is characterized by progressive hypertrophy, fibrosis, arrhythmias, heart failure and sudden cardiac death (SCD). Targeted therapy is based on enzyme replacement therapy (ERT). Recently, small molecular chaperone, migalastat, became available for patients carrying amenable pathogenic GLA variants. The management of cardiac complications requires a complex approach. Several measures differ from standard clinical guidelines. Betablockers should be used with caution due to bradycardia risk, amiodarone avoided if possible, and anticoagulation used from the first appearance of atrial fibrillation. In Fabry cardiomyopathy SCD calculators are inappropriate. The awareness of FD manifestations is essential for early identification of patients and timely treatment initiation.
\end{abstract}

Keywords: Fabry disease (FD); hypertrophic cardiomyopathy (HCM); enzyme replacement therapy (ERT); molecular chaperones

Submitted Jun 30, 2020. Accepted for publication Sep 22, 2020.

doi: $10.21037 / \mathrm{cdt}-20-593$

View this article at: http://dx.doi.org/10.21037/cdt-20-593

\section{Introduction}

Fabry disease (FD) (OMIM \# 301500) is an X-linked lysosomal storage disorder due to reduced or undetectable $\alpha$-galactosidase A (AGAL-A) enzyme activity caused by pathogenic variants in the AGAL-A gene (GLA). Tissue and organ changes are caused by widespread progressive accumulation of globotriaosylceramide $\left(\mathrm{Gb}_{3}\right)$ and its deacylated form globotriaosylsphingosine (lyso- $\left.\mathrm{Gb}_{3}\right)(1)$.

The disease is usually more severe in hemizygous males as compared to heterozygous women in whom the degree of symptoms depends on the degree of X-chromosome inactivation (XCI). The classical form of FD is a multisystemic disorder. A variety of symptoms present early, usually from childhood. However, a large proportion of patients have a late-onset phenotype manifesting predominantly as cardiac variant characterized by cardiac hypertrophy and frequently misdiagnosed as hypertrophic cardiomyopathy (HCM).

For two decades FD is being widely treated by enzyme replacement therapy (ERT) $(2,3)$. Recently, a molecular 
chaperone-migalastat was introduced for patients carrying amenable mutations in whom residual enzyme activity is effectively increased by its administration $(4,5)$.

The objective of this review is to increase the awareness of FD as the timely diagnosis is of utmost importance since targeted therapies have shown a capacity to halt or slow down the disease progression. However, their efficacy is limited in patients with irreversible organ damage.

The key questions addressed by this review is to describe typical manifestations of FD and indicate appropriate roads to timely diagnosis. In addition, we review effects of targeted therapies and indicate specific measures to be considered when treating FD-related cardiac complications. We present the following article in accordance with the NARRATIVE REVIEW reporting checklist (available at http://dx.doi.org/10.21037/cdt-20-593).

\section{Methods}

We performed a comprehensive search of recently published evidence in FD and selected most relevant papers describing larger patients' cohorts, randomized clinical trials and registry reports related to pathophysiology, clinical manifestations, diagnostic methods, treatment issues and targeted therapies.

\section{Discussion}

\section{Etiology, epidemiology, and pathophysiology}

\section{Etiology}

FD is caused by pathogenic variants in the GLA gene located on the $\mathrm{X}$ chromosome (Xq22.1). So far, over 1,000 variants distributed across the GLA gene have been reported. However, the interpretation of gene sequencing should be done with caution since several GLA variants previously reported to be disease-causing may be of uncertain significance or neutral (6).

The multisystemic early-onset disease is usually caused by variants associated with absent or very low AGAL-A enzyme levels. The late-onset (cardiac) phenotype is usually due to missense variants allowing for residual AGAL-A activity.

Female patients are usually heterozygous with a variable penetrance of the disease which may be partly explained by the process of lyonization (XCI). Females with skewed XCI in favor of the wild-type allele may be oligosymptomatic while those expressing predominantly the mutated allele may have a severe disease comparable to hemizygous males $(7,8)$.

\section{Epidemiology}

The incidence of classical FD is estimated around 1:40,000. However, newborn screening programs revealed a higher incidence of later-onset variants and detected a large number of variants of unknown significance (9). Due to founder effect some later-onset cardiac variants may be highly prevalent in countries like Taiwan (IVS4+919G>A) or Portugal (p.F113L) (10,11).

High-risk populations screening programs demonstrated that about $1 \%$ of patients originally diagnosed with HCM or unexplained left ventricular hypertrophy (LVH) are suffering from $\mathrm{FD}(12,13)$.

\section{Pathophysiology}

The storage of $\mathrm{Gb}_{3}$ occurs in various types of cells throughout the body. Lamellar storage "zebra" bodies could be found in all cardiac cells: cardiomyocytes, endothelial and vascular smooth muscle cells; valvular fibroblasts, and conduction tissue cells (Figure 1). Storage causes both structural and functional cellular impairment interfering with lysosomal functions, endocytosis and autophagy and compromising cellular energy handling. The complex interplay of disrupted cellular functions induces compensatory hypertrophy, inflammation, and ischemia leading to apoptosis, and fibrosis of the myocardium. All these changes lead to clinical manifestations, i.e., cardiac hypertrophy and replacement fibrosis, leading to signs of heart failure. The involvement of conduction system and myocardial fibrosis are responsible for various types of arrhythmias.

Since several years it became obvious that the tissue damage cannot be explained solely by $\mathrm{Gb}_{3}$ storage. Multiple studies revealed that one of the disease potentiating factors could be lyso- $\mathrm{Gb}_{3}$. It seems that lyso- $\mathrm{Gb}_{3}$ is responsible for activation of secondary mechanisms aggravating tissue damage acting namely as trophic and proinflammatory trigger (1).

While functional impairments such as energy depletion and alterations in autophagy represent a potentially reversible stage of the disease, development of interstitial and replacement myocardial fibrosis are beyond the reach of any current therapeutic interventions. Modifying the primary mechanisms by ERT or chaperone therapy at this stage may be ineffective as the disease worsening is due to mechanisms compensating for this irreversible damage $(2,3)$. 

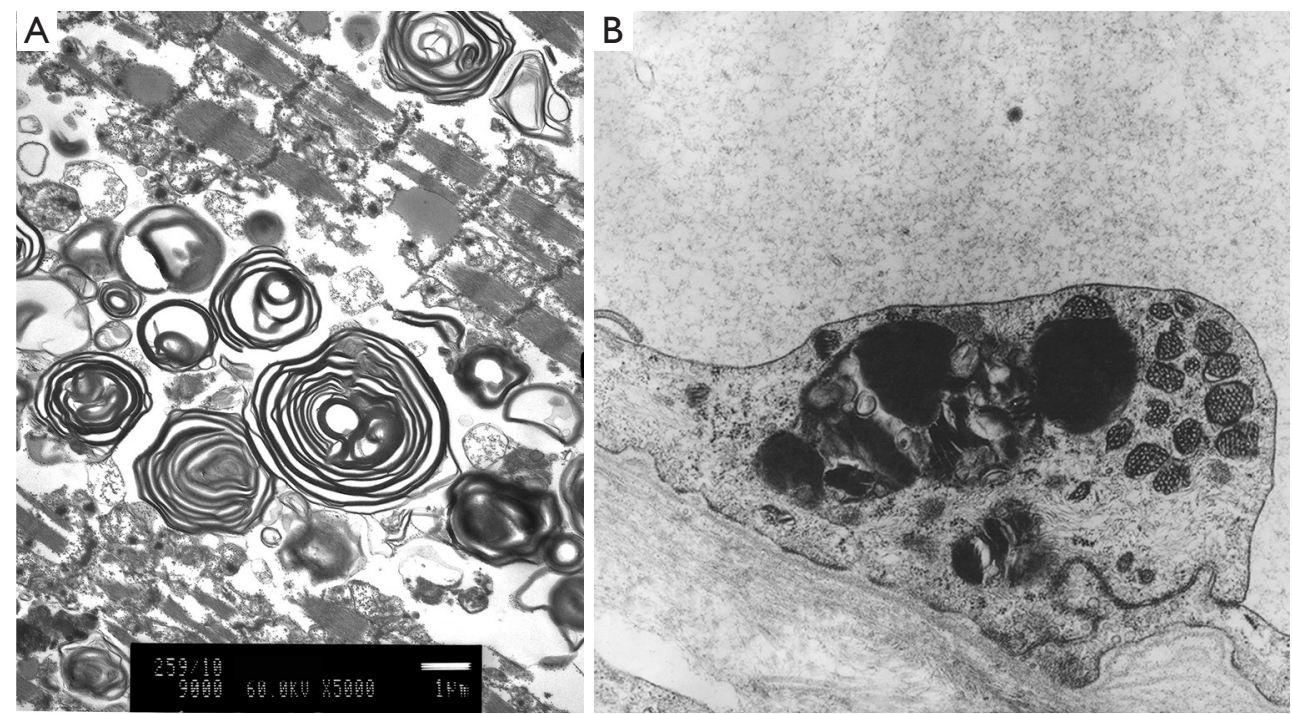

Figure 1 Histological changes in Anderson Fabry disease. Electron microscopy showing typical lamellar structures (“zebra bodies”) containing the stored substance-globotriaosylceramide $\left(\mathrm{Gb}_{3}\right)$ within a cardiomyocyte $(\mathrm{A})$ and an endothelial cell (B).

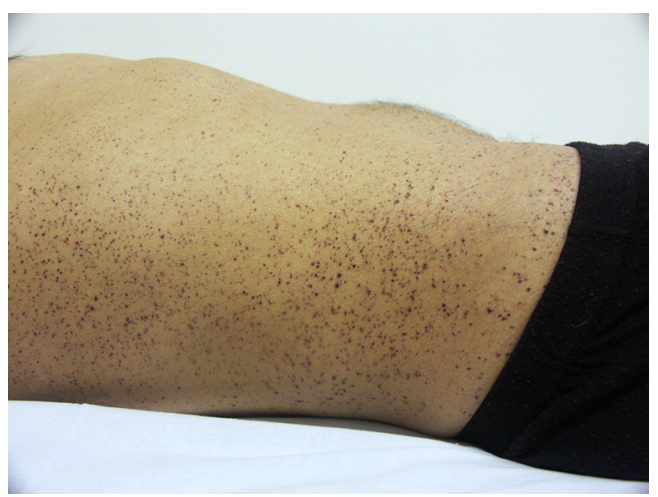

Figure 2 Angiokeratomas in a patient with classical multisystemic Anderson Fabry disease.

\section{Clinical manifestations}

\section{Clinical features of the multisystemic FD}

The multisystemic FD begins usually in early childhood by signs of painful peripheral neuropathy (acroparesthesias), reduced sweating, heat intolerance and febrile crisis. At this stage gastrointestinal symptoms are also frequent. However, these symptoms are not specific. Patients may complain about diarrhea or constipation, abdominal pain, and early satiety sensations without any clearly detectable changes on endoscopy.

Cutaneous manifestations-angiokeratomas, developing mainly in boys, represent one of the most pathognomonic signs of the disease (Figure 2). The second specific finding appearing usually in adult patients is cornea verticillataa spoke-like pattern on cornea resembling opacities seen in chronic amiodarone or chloroquine users.

Significant impairment of vital organs including the heart begins to appear usually in the third decade or later. Neurological signs include magnetic resonance imaging (MRI)-detectable white matter lesions, development of premature and often recurrent stroke, tinnitus, and hearing impairment. Renal involvement begins with microalbuminuria progressing to overt proteinuria and renal insufficiency. Development of end-stage renal disease is particularly threatening male patients (14).

\section{Cardiac manifestations}

In general, a clinically significant heart disease becomes apparent in adulthood, usually in the third or fourth decade of life in males and about ten years later in females. Later onset variants may be limited to the heart or associated with modest signs of renal disease (microalbuminuria, slight reduction of glomerular filtration rate) and appearance of cornea verticillata. The most prevalent genetic variant leading to predominant cardiac involvement is p.N215S (15).

Symptoms related to cardiac FD involvement include shortness of breath and impaired exercise tolerance, peripheral edema, palpitations, syncope, and chest pain. The underlying causes include LVH and fibrosis causing heart failure; fibrosis and conduction system involvement 


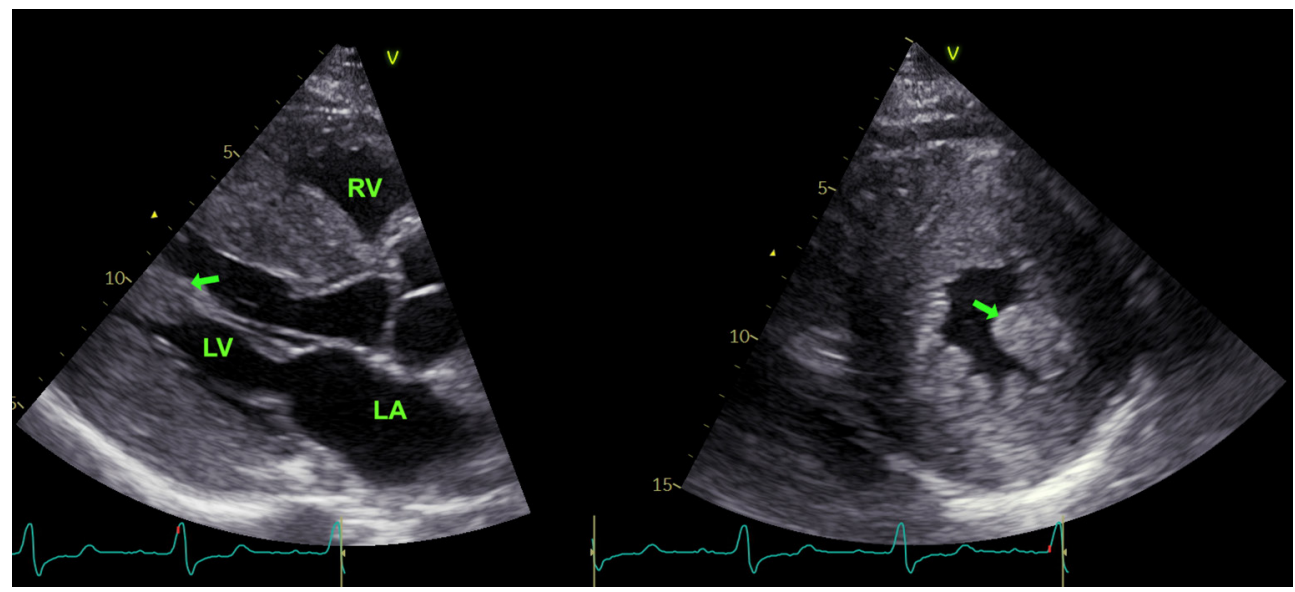

Figure 3 Echocardiography showing a diffuse character of LV hypertrophy and prominent bulging papillary muscles (arrows). LV, left ventricle; RV, right ventricle; LA, left atrium.

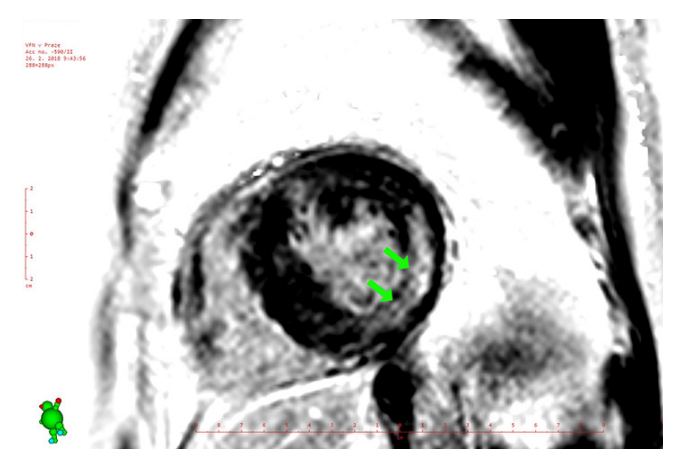

Figure 4 Cardiac MRI showing extensive late gadolinium enhancement within the posterolateral wall (arrows). MRI, magnetic resonance imaging.

triggering arrhythmias responsible for palpitations and syncope; and LVH and vascular involvement precipitating the anginal pain (16).

The most frequent appearance of cardiac disease is LVH. Its pattern is usually concentric and diffuse without left ventricular outflow tract (LVOT) obstruction (Figure 3). However, cases presenting as obstructive or apical cardiomyopathy are not exceptional. Prominent papillary muscle hypertrophy and associated right ventricular hypertrophy is seen in advanced cases. Therefore, the most frequent misdiagnosis of FD is $\operatorname{HCM}(12,13)$.

The replacement fibrosis develops predominantly within the mid-myocardial layer of the posterolateral LV segments leading to regional impairment of contractility (Figure 4) (17). Advanced stages may be associated with wall thinning and aneurysmal bulging causing secondary mitral

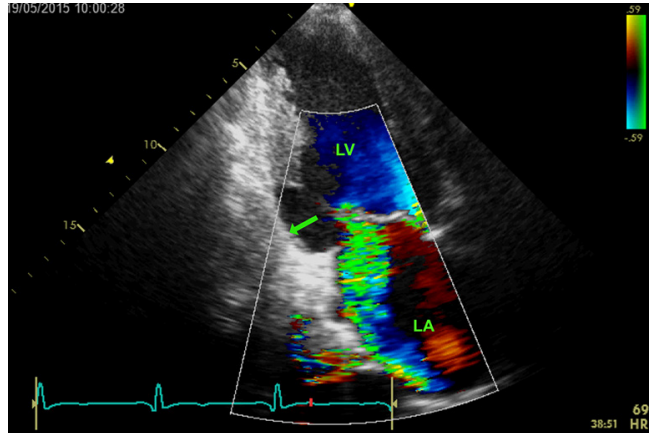

Figure 5 Echocardiography demonstrating posterior wall thinning (arrow) due to extensive scar formation and secondary mitral regurgitation. $\mathrm{LV}$, left ventricle; $\mathrm{LA}$, left atrium.

regurgitation (Figure 5).

Functional impairment associated with LVH is constant. Patients have signs of impaired LV filling while systolic function is usually preserved until very advanced stages. FD patients accumulate findings suggested as markers of heart failure with preserved ejection fraction-LVH, diastolic dysfunction by Doppler echocardiography, dilated left atrium and elevated levels of natriuretic peptides. Should the investigation of the underlying disease stop at this stage, FD would remain unrecognized (18).

Valvular disease in FD is frequent, usually characterized by mitral and aortic valvular thickening and mild regurgitations. A subset of patients presents with mitral valve prolapse (19).

Electrocardiographic (ECG) signs include a large spectrum of abnormalities (Figure 6). Development of 


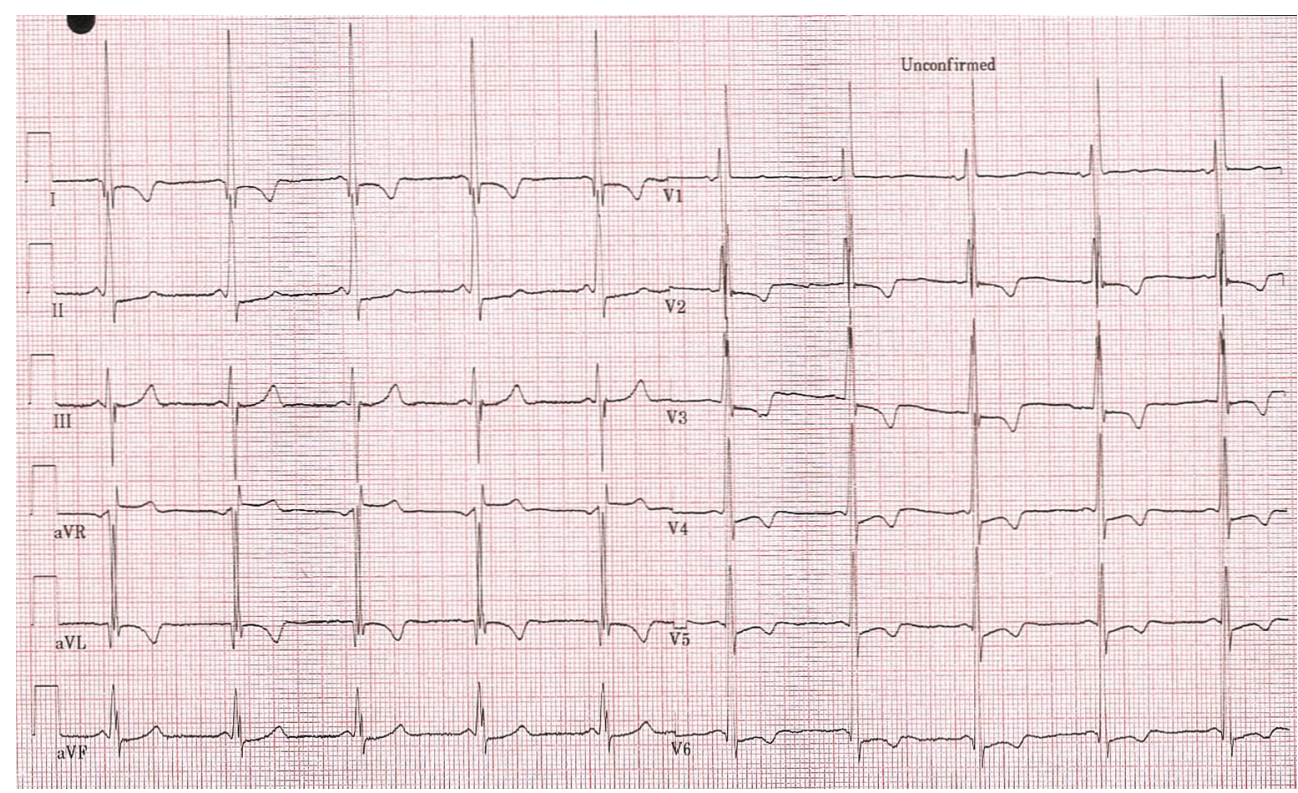

Figure 6 Typical ECG from a male FD patient showing PR interval shortening, right bundle branch pattern, signs of LV hypertrophy and marked diffuse repolarization changes. Short PR interval is not due to pre-excitation. Repolarization changes are usually present in patients with pronounced left ventricular hypertrophy and fibrosis. ECG, electrocardiography; FD, Fabry disease.

ECG signs of LVH is frequent, usually associated with extensive repolarization abnormalities. At early stage, short $\mathrm{PR}$ interval without other signs of pre-excitation is found in some patients reflecting accelerated atrioventricular (AV) conduction. Subsequently, sinus bradycardia and chronotropic incompetence may be due to sinus node or autonomic dysfunction. With progression of conduction system disease, a proportion of patients develop bundle branch and AV blocks necessitating pacemaker implantation (20). Atrial fibrillation is the most frequent arrhythmia possibly facilitated by LV diastolic dysfunction and atrial enlargement. The occurrence of ventricular arrhythmias is frequent in patients with significant LVH and fibrosis (21).

\section{Clinical investigations in Fabry cardiomyopathy}

\section{Electrocardiography}

In patients with significant FD-related cardiac disease the ECG is always abnormal, usually showing signs of $\mathrm{LVH}$ and repolarization changes (Figure 6). Conduction abnormalities include PR shortening, bundle branch or AV blocks. A proportion of patients have sinus bradycardia, atrial fibrillation or ventricular ectopy or non-sustained ventricular arrhythmias (NSVT). Prolonged Holter monitoring or loop recorders may be particularly useful for detection of clinically silent episodes of atrial fibrillation or NSVTs $(22,23)$.

\section{Echocardiography}

Echocardiography is the most accessible clinical method for $\mathrm{LVH}$ assessment. However, due to its limited reproducibility and relatively slow progression of the disease, it is not ideal for longitudinal assessments of disease progression or treatment-induced changes.

Echocardiography does not allow to establish FD diagnosis. However, a suspicion of FD may be expressed particularly in presence of diffuse LVH with prominent papillary muscles or right ventricular hypertrophy, or in presence of large scars of the posterolateral wall $(24,25)$. The use of speckle tracking strain analysis may help to reveal impairment of deformation within the areas affected by replacement fibrosis (26). Another situation potentially rising a suspicion of FD is the presence of $\mathrm{LVH}$ and mitral valve prolapse. In contrast, the "binary sign" described in the past as one of typical features of FD-related cardiomyopathy is not specific (27).

Echocardiography allows evaluation of left ventricular (LV) filling by a combination of transmitral flow pattern and tissue Doppler measurements and evaluation of left 
atrial dilatation. Available data suggest that similarly to other types of LVH, FD cardiomyopathy is associated with diastolic dysfunction (18). However, compared to amyloidosis, the prevalence of restrictive physiology is rare and limited to advanced cases (28). Pulmonary hypertension in FD is usually mild and does not explain the frequently seen right ventricular hypertrophy (25).

Echocardiography also allows the assessment of other FD-related changes including left heart valvular thickenings, mitral valve prolapse or aortic bulb and ascending aorta dilatation (19).

\section{Cardiac magnetic resonance}

Cardiac magnetic resonance provides a more accurate and reproducible assessments of cardiac structures. It also allows tissue characterization. Late gadolinium enhancement (LGE) revealed first the nature of fibrotic changes in FD cardiomyopathy (29). Our understanding of the processes was further refined by the use of $\mathrm{T} 1$ mapping. The low native $\mathrm{T} 1$ is highly specific for FD cardiomyopathy possibly reflecting the increased lipid content within the myocardium $(30,31)$. In contrast, the pseudo-normalization of T1 within posterolateral wall suggests the development of fibrosis. Recently, the use of T2 mapping pointed out the possible inflammatory process leading to fibrotic changes within this area (32).

\section{Nuclear perfusion imaging}

Although perfusion imaging [either by single photon emission computed tomography (SPECT) or positron emission tomography (PET)] is not representing a routine part of cardiac assessments in FD, it improved our understanding of its pathophysiology. It was repeatedly shown that FD patients have a decreased coronary flow reserve, possibly due to microvascular involvement. Of note, the impairment of coronary perfusion was seen even in absence of LVH suggesting that ischemia may be one of the underappreciated triggers of initial myocardial damage in FD $(33,34)$. In addition, many FD patients develop stenotic lesions of major coronary arteries (35).

\section{Biomarkers}

The use of biomarkers in FD is useful for diagnostic purposes and prognostic stratification as well.

Lyso- $\mathrm{Gb}_{3}$ is considered the most useful biochemical parameter reflecting FD severity. The higher values are seen in genetic variants associated with the classic multisystemic disease, particularly in male patients. Cardiac variants are associated with significantly lower values. However, lyso$\mathrm{Gb}_{3}$ assessment in the presence of a variant of unknown significance is particularly useful. In case of normal or minimally elevated lyso- $\mathrm{Gb}_{3}$ the pathogenicity of the mutation should be doubted (36,37).

Another use of lyso- $\mathrm{Gb}_{3}$ is evaluation of treatment responses to ERT or migalastat. The lack of reduction or even an increase of lyso- $\mathrm{Gb}_{3}$ during the treatment may suggest development of antibodies in the former and limited amenability in the latter.

Cardiac markers such as natriuretic peptides and troponins may help to monitor the disease progression. Natriuretic peptides were shown to correlate with the degree of LV diastolic dysfunction and heart failure severity (38). The presence of high-sensitivity troponin increase is usually signaling a severe disease with fibrosis of the myocardium and unfavorable prognosis (39).

\section{Endomyocardial biopsy}

In most cases of FD cardiomyopathy endomyocardial biopsy is not necessary. The diagnosis can be readily made on the basis of imaging results, genetic testing and lyso- $\mathrm{Gb}_{3}$ analysis. It may be considered in exceptional cases with variant of unknown significance, high residual enzyme activity (typically above $10 \%$ ) and low lyso-Gb However, even benign genetic variants may be associated with minimal storage showing scarce "zebra bodies" on electron microscopy. Therefore, an evaluation by an expert pathologist is always mandated (40).

\section{Diagnosis of FD}

\section{Strategies of FD diagnosis}

The pathways to diagnosis differ according to identification of the index case. The easiest way is the cascade family screening based on gene sequencing after identifying a proband. In other situations, patients are being referred for laboratory (enzyme activity, lyso- $\mathrm{Gb}_{3}$ ) and gene testing based on their signs and symptoms.

Many patients are being diagnosed within high-risk populations screening programs (e.g., in patients with HCM, end-stage renal disease, stroke etc.). Nationwide or regional newborn screening programs are also performed $(9,10)$.

Different strategies are used for identification of patients. In most programs, enzyme activity is being tested from leukocytes or plasma. Dry blood spot testing is often applied in large scale screening programs (41). Assessment 
of enzymatic activity seems sensitive enough in male patients in whom it is always severely reduced as compared to healthy individuals (42). However, this approach fails in women in whom enzymatic activity may be overlapping with normal ranges. Therefore, some laboratories are using stratification by lyso- $\mathrm{Gb}_{3}$ measurements, others are proceeding directly to gene testing in all females $(43,44)$.

\section{Gene sequencing}

Gene sequencing is mandatory in all suspected FD patients, particularly before initiation of any targeted therapy. It has been suggested that all adult patients with unexplained LVH should undergo a testing for FD. Subsequently, GLA was incorporated to many panels used with high throughput next generation sequencing for screening in high-risk patients including those with HCM. This has resulted in identification of many GLA variants of unknown significance. Therefore, an expert assessment of identified variants is necessary to exclude non-disease-causing variants in whom another cause of their disease should be investigated (44).

\section{Clinical management}

\section{ERT}

ERT represents a standard of FD treatment already for two decades. Two preparations-agalsidase alfa and beta-are widely used in EU. The treatment has been shown effective in slowing renal disease progression and improving several symptoms of the disease (45). However, despite many efforts a robust proof about preventability of cardiac complications and survival benefits of ERT is still vague (46). Although ERT has shown a modest reduction of cardiac mass, data about preventability of fibrosis are lacking. Moreover, only limited evidence exists about improvements of symptoms and cardiac complications $(47,48)$. This is mainly due to the slow progression of FD. Demonstration of significant benefits would require a long-term detailed follow-up which is not achievable in any randomized controlled trial.

Beneficial effects of ERT were confirmed by analyses of large international registries (Fabry Registry, Fabry Outcome Survey) (49). In contrast, some authors were unable to show a survival benefit with ERT in their cohorts (50). However, early results were obscured by inclusion of very advanced cases with irreversible organ damage in whom the ERT has little if any impact on outcomes. Nowadays, ERT is being regarded rather as a preventative therapy to be given early in the course of the disease (51).

A proportion of patients develops antibodies against the infused enzyme (52). Despite an emerging evidence of clinical impacts of this adverse reaction, an appropriate management does not exist (53). It seems that at least in some cases the antibodies may be saturated by administering an excess of the enzyme (54).

\section{Migalastat}

A small molecular chaperone migalastat was approved for the treatment of FD in EU since 2016 and in USA since 2018. The chaperone is effective only in cases with residual enzyme activity which is increased by its administration. This is related to the underlying gene variant described as "amenable" mutations (4). The main argument for migalastat introduction on the market was the achievement of significant $\mathrm{LV}$ mass reduction in two independent trials (55). Their results were confirmed by a recently published analysis of a multicenter trial "FAMOUS" from Germany. However, its authors put in question amenability of several mutations listed originally as amenable by the drug producer. The trial also raises a concern about migalastat impacts on glomerular filtration rate decrease rate (56).

\section{Therapies in development}

Further development is aiming to improve the already approved strategy of ERT by ameliorated enzymes. Pegunigalsidase alfa is a chemically modified pegylated covalently cross-liked enzyme produced by a plant-based system. The enzyme has a substantially prolonged plasma half-life and low immunogenicity. Pegunigalsidase alfa is already in advanced stages of clinical development and testing (57). In contrast, another enzyme derived from moss is at the beginning of clinical investigations (58).

Another approach is based on substrate reduction by inhibition of glucosylceramide synthase. Two oral preparations-lucerastat and venglustat-are being tested in clinical settings after demonstrating promising effects in preclinical trials (59).

In addition, several gene therapies are being extensively tested using either in-vivo or ex-vivo gene transfer with different viral vectors (60).

\section{Management of cardiac complications}

The management of cardiac complications in FD patients usually follow the internationally validated guidelines. However, some differences should be emphasized. Several 
drugs should be used with caution, such as verapamil, betablockers and ivabradine which may aggravate the chronotropic incompetence or impair AV conduction. Amiodarone should be avoided as much as possible since it has a potential to interfere with lysosomal functions (61).

Anticoagulation in atrial fibrillation related to FD should not be guided by currently proposed scoring systems. Instead, FD patients should be considered at high risk for systemic embolism and anticoagulated properly whenever atrial fibrillation develops. Although firm data are lacking, the use of direct anticoagulants may be of advantage as FD also increases risks of cerebral bleeding. Moreover, as compared to warfarin direct anticoagulants have potentially less pronounced effects on renal disease progression (62).

Sudden cardiac death estimates proposed in HCM are not applicable in FD. From the published literature it seems that patients with advanced disease with $\mathrm{LVH}$, fibrosis, history of syncope or NSVTs are at higher risk and should be considered for implantable cardioverter defibrillator (ICD) implantation (63).

\section{Limitations of available evidence}

The understanding of pathophysiology of FD is constantly evolving, namely due to appearance of novel biomarkers such as lyso- $\mathrm{Gb}_{3}$ or imaging techniques such as magnetic resonance T1 mapping. Since FD is a rare disease, most data available are limited by a selection bias of small cohorts described in the literature. Larger datasets are generated by international registries primarily designed to monitor ERT results. However, these data are subject to selection bias as well. Moreover, longitudinal follow-up within registries is usually limited by incomplete data reporting. This results in vague evidence of survival benefits and limited availability of data demonstrating improvements in cardiac complications achieved by current targeted therapies. Further research should focus on bridging the gaps in ERT efficacy evidence, analysis of antibody response to ERT, and on development of novel therapeutic strategies.

\section{Summary}

FD, particularly its later-onset phenotype, is a relatively frequent finding among patients originally diagnosed with HCM. Targeted therapies have a potential to change the course of the disease provided they are started before irreversible damage develops. This increases the importance of timely diagnosis of the probands and their family members. Since systemic signs of FD may be absent in lateonset cardiac variants, all adult patients with unexplained LVH should be tested for FD.

Even in advanced cases the appropriate management of cardiac complications may alleviate the symptoms and prolong the life of our patients. The essential measures include heart failure treatments, arrhythmia management including anticoagulation in atrial fibrillation and ICD implantation in high-risk patients.

Development of novel therapies is usually based on evaluation of surrogate endpoints such as cardiac mass, glomerular filtration rate, gastrointestinal symptoms, or pain. Once approved for clinical use they would require an international collaboration to gather large clinical datasets able to confirm their effects on survival and clinical complications.

\section{Acknowledgments}

Funding: Supported by PROGRES Q38-an institutional support for Charles University.

\section{Footnote}

Provenance and Peer Review: This article was commissioned by the Guest Editors (Yskert von Kodolitsch, Harald Kaemmerer, Koichiro Niwa) for the series "Current Management Aspects in Adult Congenital Heart Disease (ACHD): Part III" published in Cardiovascular Diagnosis and Therapy. The article has undergone external peer review.

Reporting Checklist: The authors have completed the NARRATIVE REVIEW reporting checklist. Available at http://dx.doi.org/10.21037/cdt-20-593

Conflicts of Interest: Both authors have completed the ICMJE uniform disclosure form (available at http:// dx.doi.org/10.21037/cdt-20-593). The series "Current Management Aspects in Adult Congenital Heart Disease (ACHD): Part III" was commissioned by the editorial office without any funding or sponsorship. AL reports other from Sanofi genzyme, other from Takeda, during the conduct of the study; personal fees from Sanofi Genzyme, personal fees from Takeda, outside the submitted work. TP reports other from Sanofi genzyme, other from Takeda, during the conduct of the study. 
Ethical Statement: The authors are accountable for all aspects of the work in ensuring that questions related to the accuracy or integrity of any part of the work are appropriately investigated and resolved.

Open Access Statement: This is an Open Access article distributed in accordance with the Creative Commons Attribution-NonCommercial-NoDerivs 4.0 International License (CC BY-NC-ND 4.0), which permits the noncommercial replication and distribution of the article with the strict proviso that no changes or edits are made and the original work is properly cited (including links to both the formal publication through the relevant DOI and the license). See: https://creativecommons.org/licenses/by-nc-nd/4.0/.

\section{References}

1. Aerts JM, Groener JE, Kuiper S, et al. Elevated globotriaosylsphingosine is a hallmark of Fabry disease. Proc Natl Acad Sci U S A 2008;105:2812-7.

2. Banikazemi M, Bultas J, Waldek S, et al. Agalsidase-beta therapy for advanced Fabry disease: a randomized trial. Ann Intern Med 2007;146:77-86.

3. Schiffmann R, Kopp JB, Austin HA 3rd, et al. Enzyme replacement therapy in Fabry disease: a randomized controlled trial. JAMA 2001;285:2743-9.

4. Benjamin ER, Della Valle MC, $\mathrm{Wu} X$, et al. The validation of pharmacogenetics for the identification of Fabry patients to be treated with migalastat. Genet Med 2017;19:430-8.

5. Germain DP, Hughes DA, Nicholls K, et al. Treatment of Fabry's Disease with the Pharmacologic Chaperone Migalastat. N Engl J Med 2016;375:545-55.

6. Smid BE, van der Tol L, Cecchi F, et al. Uncertain diagnosis of Fabry disease: consensus recommendation on diagnosis in adults with left ventricular hypertrophy and genetic variants of unknown significance. Int J Cardiol 2014;177:400-8.

7. Dobrovolny R, Dvorakova L, Ledvinova J, et al. Relationship between $\mathrm{X}$-inactivation and clinical involvement in Fabry heterozygotes. Eleven novel mutations in the alpha-galactosidase A gene in the Czech and Slovak population. J Mol Med (Berl) 2005;83:647-54.

8. Echevarria L, Benistan K, Toussaint A, et al. $\mathrm{X}$-chromosome inactivation in female patients with Fabry disease. Clin Genet 2016;89:44-54.

9. Spada M, Pagliardini S, Yasuda $M$, et al. High incidence of later-onset fabry disease revealed by newborn screening.
Am J Hum Genet 2006;79:31-40.

10. Lin HY, Chong KW, Hsu JH, et al. High incidence of the cardiac variant of Fabry disease revealed by newborn screening in the Taiwan Chinese population. Circ Cardiovasc Genet 2009;2:450-6.

11. Azevedo O, Gal A, Faria R, et al. Founder effect of Fabry disease due to p.F113L mutation: Clinical profile of a lateonset phenotype. Mol Genet Metab 2020;129:150-60.

12. Doheny D, Srinivasan R, Pagant S, et al. Fabry Disease: prevalence of affected males and heterozygotes with pathogenic GLA mutations identified by screening renal, cardiac and stroke clinics, 1995-2017. J Med Genet 2018;55:261-8.

13. Elliott P, Baker R, Pasquale F, et al. Prevalence of Anderson-Fabry disease in patients with hypertrophic cardiomyopathy: the European Anderson-Fabry Disease survey. Heart 2011;97:1957-60.

14. Talbot AS, Lewis NT, Nicholls KM. Cardiovascular outcomes in Fabry disease are linked to severity of chronic kidney disease. Heart 2015;101:287-93.

15. Germain DP, Brand E, Burlina A, et al. Phenotypic characteristics of the p.Asn215Ser (p.N215S) GLA mutation in male and female patients with Fabry disease: A multicenter Fabry Registry study. Mol Genet Genomic Med 2018;6:492-503.

16. Linhart A, Kampmann C, Zamorano JL, et al. Cardiac manifestations of Anderson-Fabry disease: results from the international Fabry outcome survey. Eur Heart J 2007;28:1228-35.

17. Moon JC, Sheppard M, Reed E, et al. The histological basis of late gadolinium enhancement cardiovascular magnetic resonance in a patient with Anderson-Fabry disease. J Cardiovasc Magn Reson 2006;8:479-82.

18. Linhart A, Cecchi F. Common presentation of rare diseases: Left ventricular hypertrophy and diastolic dysfunction. Int J Cardiol 2018;257:344-50.

19. Linhart A, Palecek T, Bultas J, et al. New insights in cardiac structural changes in patients with Fabry's disease. Am Heart J 2000;139:1101-8.

20. O'Mahony C, Coats C, Cardona M, et al. Incidence and predictors of anti-bradycardia pacing in patients with Anderson-Fabry disease. Europace 2011;13:1781-8.

21. Krämer J, Niemann $M$, Störk S, et al. Relation of burden of myocardial fibrosis to malignant ventricular arrhythmias and outcomes in Fabry disease. Am J Cardiol 2014;114:895-900.

22. Niemann M, Hartmann T, Namdar M, et al. Crosssectional baseline analysis of electrocardiography in a large 
cohort of patients with untreated Fabry disease. J Inherit Metab Dis 2013;36:873-9.

23. Weidemann F, Maier SK, Störk S, et al. Usefulness of an Implantable Loop Recorder to Detect Clinically Relevant Arrhythmias in Patients With Advanced Fabry Cardiomyopathy. Am J Cardiol 2016;118:264-74.

24. Niemann M, Liu D, Hu K, et al. Prominent papillary muscles in Fabry disease: a diagnostic marker? Ultrasound Med Biol 2011;37:37-43.

25. Palecek T, Dostalova G, Kuchynka P, et al. Right ventricular involvement in Fabry disease. J Am Soc Echocardiogr 2008;21:1265-8.

26. Krämer J, Niemann M, Liu D, et al. Two-dimensional speckle tracking as a non-invasive tool for identification of myocardial fibrosis in Fabry disease. Eur Heart J 2013;34:1587-96.

27. Mundigler G, Gaggl M, Heinze G, et al. The endocardial binary appearance ('binary sign') is an unreliable marker for echocardiographic detection of Fabry disease in patients with left ventricular hypertrophy. Eur J Echocardiogr 2011;12:744-9.

28. Marek J, Palecek T, Magne J, et al. Comparison of echocardiographic parameters in Fabry cardiomyopathy and light-chain cardiac amyloidosis. Echocardiography 2018;35:1755-63

29. Moon JC, Sachdev B, Elkington AG, et al. Gadolinium enhanced cardiovascular magnetic resonance in Anderson-Fabry disease. Evidence for a disease specific abnormality of the myocardial interstitium. Eur Heart J 2003;24:2151-5.

30. Sado DM, White SK, Piechnik SK, et al. Identification and assessment of Anderson-Fabry disease by cardiovascular magnetic resonance noncontrast myocardial T1 mapping. Circ Cardiovasc Imaging 2013;6:392-8.

31. Thompson RB, Chow K, Khan A, et al. T1 mapping with cardiovascular MRI is highly sensitive for Fabry disease independent of hypertrophy and sex. Circ Cardiovasc Imaging 2013;6:637-45.

32. Nordin S, Kozor R, Medina-Menacho K, et al. Proposed Stages of Myocardial Phenotype Development in Fabry Disease. JACC Cardiovasc Imaging 2019;12:1673-83.

33. Elliott PM, Kindler H, Shah JS, et al. Coronary microvascular dysfunction in male patients with AndersonFabry disease and the effect of treatment with alpha galactosidase A. Heart 2006;92:357-60.

34. Tomberli B, Cecchi F, Sciagrà R, et al. Coronary microvascular dysfunction is an early feature of cardiac involvement in patients with Anderson-Fabry disease. Eur
J Heart Fail 2013;15:1363-73.

35. Kovarnik T, Mintz GS, Karetova D, et al. Intravascular ultrasound assessment of coronary artery involvement in Fabry disease. J Inherit Metab Dis 2008;31:753-60.

36. Ferreira S, Auray-Blais C, Boutin M, et al. Variations in the GLA gene correlate with globotriaosylceramide and globotriaosylsphingosine analog levels in urine and plasma. Clin Chim Acta 2015;447:96-104.

37. Nowak A, Mechtler T, Kasper DC, et al. Correlation of Lyso-Gb3 levels in dried blood spots and sera from patients with classic and Later-Onset Fabry disease. Mol Genet Metab 2017;121:320-4.

38. Coats CJ, Parisi V, Ramos M, et al. Role of serum $\mathrm{N}$-terminal pro-brain natriuretic peptide measurement in diagnosis of cardiac involvement in patients with andersonfabry disease. Am J Cardiol 2013;111:111-7.

39. Seydelmann N, Liu D, Krämer J, et al. High-Sensitivity Troponin: A Clinical Blood Biomarker for Staging Cardiomyopathy in Fabry Disease. J Am Heart Assoc 2016;5:e002839.

40. Hsu TR, Sung SH, Chang FP, et al. Endomyocardial biopsies in patients with left ventricular hypertrophy and a common Chinese later-onset Fabry mutation (IVS4 + 919G > A). Orphanet J Rare Dis 2014;9:96.

41. Reuser AJ, Verheijen FW, Bali D, et al. The use of dried blood spot samples in the diagnosis of lysosomal storage disorders--current status and perspectives. Mol Genet Metab 2011;104:144-8.

42. Desnick RJ, Desnick SJ, Raman MK, et al. Fabry's disease: enzymatic diagnosis of hemizygotes and heterozygotes. Alpha-galactosidase activities in plasma, serum, urine, and leukocytes. J Lab Clin Med 1973;81:157-71.

43. Rombach SM, Dekker N, Bouwman MG, et al. Plasma globotriaosylsphingosine: diagnostic value and relation to clinical manifestations of Fabry disease. Biochim Biophys Acta 2010;1802:741-8.

44. Ortiz A, Germain DP, Desnick RJ, et al. Fabry disease revisited: Management and treatment recommendations for adult patients. Mol Genet Metab 2018;123:416-27.

45. Germain DP, Waldek S, Banikazemi M, et al. Sustained, long-term renal stabilization after 54 months of agalsidase beta therapy in patients with Fabry disease. J Am Soc Nephrol 2007;18:1547-57.

46. Germain DP, Charrow J, Desnick RJ, et al. Ten-year outcome of enzyme replacement therapy with agalsidase beta in patients with Fabry disease. J Med Genet 2015;52:353-8.

47. Lobo T, Bjorksten JM, Nicholls K, et al. Cardiovascular 
testing in Fabry disease: exercise capacity reduction, chronotropic incompetence and improved anaerobic threshold after enzyme replacement. Intern Med J 2008;38:407-14.

48. Kampmann C, Linhart A, Devereux RB, et al. Effect of agalsidase alfa replacement therapy on Fabry diseaserelated hypertrophic cardiomyopathy: a 12- to 36-month, retrospective, blinded echocardiographic pooled analysis. Clin Ther 2009;31:1966-76.

49. Ortiz A, Abiose A, Bichet DG, et al. Time to treatment benefit for adult patients with Fabry disease receiving agalsidase beta: data from the Fabry Registry. J Med Genet 2016;53:495-502.

50. Weidemann F, Niemann M, Störk S, et al. Long-term outcome of enzyme-replacement therapy in advanced Fabry disease: evidence for disease progression towards serious complications. J Intern Med 2013;274:331-41.

51. Weidemann F, Niemann M, Breuning F, et al. Longterm effects of enzyme replacement therapy on fabry cardiomyopathy: evidence for a better outcome with early treatment. Circulation 2009;119:524-9.

52. Lenders M, Brand E. Effects of Enzyme Replacement Therapy and Antidrug Antibodies in Patients with Fabry Disease. J Am Soc Nephrol 2018;29:2265-78.

53. Stappers F, Scharmetzki D, Schmitz D, et al. Neutralising anti-drug antibodies in Fabry disease can inhibit endothelial enzyme uptake and activity. J Inherit Metab Dis 2020;43:334-47.

54. Lenders M, Neusser LP, Rudnicki M, et al. DoseDependent Effect of Enzyme Replacement Therapy on Neutralizing Antidrug Antibody Titers and Clinical Outcome in Patients with Fabry Disease. J Am Soc Nephrol 2018;29:2879-89.

55. Hughes DA, Nicholls K, Shankar SP, et al. Oral pharmacological chaperone migalastat compared with

Cite this article as: Linhart A, Paleček T. Narrative review on Morbus Fabry: diagnosis and management of cardiac manifestations. Cardiovasc Diagn Ther 2021;11(2):650-660. doi: $10.21037 / \mathrm{cdt}-20-593$ enzyme replacement therapy in Fabry disease: 18-month results from the randomised phase III ATTRACT study. J Med Genet 2017;54:288-96.

56. Lenders M, Nordbeck P, Kurschat C, et al. Treatment of Fabry's Disease With Migalastat: Outcome From a Prospective Observational Multicenter Study (FAMOUS). Clin Pharmacol Ther 2020;108:326-37.

57. Schiffmann R, Goker-Alpan O, Holida M, et al. Pegunigalsidase alfa, a novel PEGylated enzyme replacement therapy for Fabry disease, provides sustained plasma concentrations and favorable pharmacodynamics: A 1-year Phase 1/2 clinical trial. J Inherit Metab Dis 2019;42:534-44.

58. Shen JS, Busch A, Day TS, et al. Mannose receptormediated delivery of moss-made alpha-galactosidase A efficiently corrects enzyme deficiency in Fabry mice. J Inherit Metab Dis 2016;39:293-303.

59. Ashe KM, Budman E, Bangari DS, et al. Efficacy of Enzyme and Substrate Reduction Therapy with a Novel Antagonist of Glucosylceramide Synthase for Fabry Disease. Mol Med 2015;21:389-99.

60. Simonetta I, Tuttolomondo A, Di Chiara T, et al. Genetics and Gene Therapy of Anderson-Fabry Disease. Curr Gene Ther 2018;18:96-106.

61. Ikeda K, Hirayama M, Hirota Y, et al. Drug-induced phospholipidosis is caused by blockade of mannose 6-phosphate receptor-mediated targeting of lysosomal enzymes. Biochem Biophys Res Commun 2008;377:268-74.

62. Connolly SJ, Ezekowitz MD, Yusuf S, et al. Dabigatran versus warfarin in patients with atrial fibrillation. $\mathrm{N}$ Engl J Med 2009;361:1139-51.

63. Baig S, Edward NC, Kotecha D, et al. Ventricular arrhythmia and sudden cardiac death in Fabry disease: a systematic review of risk factors in clinical practice. Europace 2018;20:f153-61. 